\title{
Editorial: Revolution?!
}

\author{
Daniel KÜNZLER', Sebastian SCHIEF ${ }^{1}$
}

\section{Liebe Leserinnen und Leser}

Revolutionen brechen scheinbar unvermittelt in den Gang der Geschichte ein. Ein Bespiel ist die Serie von Protesten, Aufständen und Revolutionen in der arabischen Welt, die im Dezember 2010 begann und als "Arabischer Frühling» bekannt wurde. Mit atemberaubender Geschwindigkeit brachen Machtstrukturen ein, die zuvor scheinbar unüberwindbar schienen. Die Sozialwissenschaften haben Mühe, derartige Ereignisse zu prognostizieren und begnügen sich mit ex-post-Erklärungen, die möglicherweise eine künstliche Kohärenz in eine Ereignisfolge konstruieren. Eine ganze Reihe von Wissenschaftlern und Wissenschaftlerinnen haben sich mit Revolutionen befasst (z.B. Dahrendorf 1990, Eisenstadt 1978, Huntington 1968, Kuran 1991, Marx und Engels 1848, Pareto 1968, Skocpol 1979, Smelser 1959, Sorokin 1925, Tilly 1996, Weber 1995). Politiker und Politikerinnen und andere Persönlichkeiten wiederum haben deren Realisierung angestrebt (z.B. Bolívar, Engels, Guevara, Lenin, Luxemburg, Mao Zedong, Marx oder Popovic). Während eine erste Generation von Arbeiten sich hauptsächlich deskriptiv mit dem historischen Verlauf von Revolutionen auseinandergesetzt hat, dominierten modernisierungstheoretische Perspektiven die zweite Generation und marxistische Perspektiven die dritte (Goldstone 1982; 1986; siehe auch den Beitrag von Sheikhzadegan in dieser Ausgabe). Eine vierte Generation hat dann auf die Bedeutung von Kultur und Ideologie verwiesen (Goldstone 2001).

In der Regel werden verschiedene Arten von Revolutionen unterschieden (siehe z.B. Grosser 2013). Gängig ist beispielsweise eine Unterscheidung nach den Grundwerten, welche von Revolutionen vertreten werden: Gleichheit (sozialistische Revolutionen, siehe den Beitrag von Künzler), Freiheit (bürgerliche Revolutionen) oder deren Ablehnung (konservativantimodernistische Revolutionen, siehe den Beitrag von Sheikhzadegan). Der Begriff der Revolution bezieht sich aber nicht nur auf derartige mehr oder weniger gewaltsame abrupte Veränderungen der sozialen und politischen Ordnung, sondern auch auf eher graduelle Umwälzungen wirtschaftlicher und sozialer, technologischer oder kulturell-ideologischer Art. Als wirtschaftliche und soziale Revolutionen diskutiert werden in dieser Ausgabe beispielsweise die erste (z.B. Landes 1983), zweite (z.B. Friedmann 1936), dritte (z.B. Bell 1990 oder Rifkin 1995) und vierte (siehe den Beitrag von Flecker) industrielle Revolution. Im Bereich der technologischen Revolutionen werden etwa die neolithische Revolution (z.B. Childe 1951, Lenski et al. 1991 oder Sanderson 1999), die grüne Revolution (Gaud 1968) oder die digitale Revolution (siehe den Beitrag von $\mathrm{Kahl}$ ) diskutiert. $\mathrm{Zu}$ den kulturell-ideologischen Revolutionen gehören etwa die Kulturrevolution (Kraus 2012), die sexuelle Revolution (Allyn 2000) oder

\footnotetext{
${ }^{1}$ Departement für Sozialwissenschaften, Universität Fribourg
} 
die wissenschaftlichen Revolutionen (Kuhn 2006). Allerdings gibt es auch starke Diskontinuitäten, die nicht als Revolutionen erfasst werden.

Das Schwerpunktheft Revolution?! macht sich diesen sehr breiten Revolutionsbegriff zu Eigen und behandelt ganz unterschiedliche soziale Phänomene, die unter dem Begriff Revolution eingeordnet werden können, respektive - wie das Fragezeichen andeutet - oftmals sozialwissenschaftlich oder umgangssprachlich als Revolutionen apostrophiert werden. Die Bandbreite umfasst Massenüberwachung und die Sammlung von Daten $(K a h l)$, die Energiewende in Deutschland (Neukirch), die Digitalisierung der Arbeitswelt (Flecker), die von Keynes prognostizierte aber ausgebliebene radikale Arbeitszeitverkürzung (Schief) sowie die politischen Revolutionen im engeren Sinne in Iran (Sheikhzadegan) und auf den Seychellen (Künzler).

Der erste Beitrag von Martin Kahl unter dem Titel Big Data-Revolution, Überwachung und soziale Medien: Gefahr für die Demokratie? diskutiert die Folgen der Umwälzungen im Bereich der Datenspeicherung für unser politisches System. Unternehmen, Sicherheitsbehörden und politische Organisationen sammeln und verwerten eine Unmenge persönlicher Daten mit dem Argument der Verbesserung von Verhaltensprognosen und zur Verhaltenssteuerung. Setzt man diese Entwicklung mit Kernelementen demokratischer Willensbildung wie der autonomen Meinungsbildung, der selbstbestimmten Grenzziehung zwischen Privatheit und Öffentlichkeit und der Vermittlungsaufgabe öffentlicher Medien in Beziehung, so werden an vielen Stellen tiefgreifende Veränderungen und Probleme deutlich. Die gesellschaftlichen und politischen Folgen der massenhaften Erhebung und Auswertung persönlicher Daten sind aus demokratietheoretischer Perspektive jedoch erst in Ansätzen behandelt worden. Kahls Beitrag illustriert gegenwärtige Überwachungspraktiken und erörtert ihre komplexen, teilweise gegenläufigen Folgen mit Blick auf die Grundbedingungen demokratischer Willensbildung.

Mario Neukirch nimmt sich in seinem Beitrag Die Energiewende in der Bundesrepublik Deutschland (1974-2017) - Reform, Revolution, oder Restauration? der fundamentalen Veränderungen in der Energiepolitik der Bundesrepublik Deutschland an. Sein Artikel untersucht die Transformation des deutschen Elektrizitätsversorgungssystems im Zeitraum 1974-2017 aus einer Makroperspektive. Dabei wird diese Transformation als Konflikt zweier Grosskoalitionen betrachtet: Etablierte, die den Status quo verteidigen und Herausforderer, von denen die Energiewende gefordert bzw. technisch und wirtschaftlich umgesetzt wird. Der Analysezeitraum wird in drei zeitlichen Phasen betrachtet. In der ersten Phase diskursiver Konfrontation fordert eine Graswurzelkoalition Staat und Energiekonzerne heraus, kann aber die quasi revolutionären Forderungen u.a. nach einer Demokratisierung des Energiesystems nicht durchsetzen. Ermöglicht durch eine weitgehende Restrukturierung beider Koalitionen in Phase zwei, kommt es seit Ende der 80er Jahre zu einer partiellen Umsetzung der Ideen. In der 2011 beginnenden dritten Phase schliesslich, gelingt es der etablierten Koalition, die z. T. verlorene Kontrolle über das Energiesystem zurückzugewinnen.

Jörg Flecker nimmt sich in seinem Betrag TINA und die technologische Revolution dem immer populärer werdenden Thema der Digitalisierung der Arbeit oder auch der Industrie 4.0 an. In seinem Beitrag geht es darum aufzuzeigen, dass die Verschränkung der als linear und notwendig gedeuteten Technikentwicklung im Bereich der Informations- und Kommunikationstechnologien mit einem neoliberalen gesellschaftspolitischen Programm sich insbe- 
sondere in folgenden drei Dimensionen fassen lässt: Der einseitig kapital- und managementorientierten Gestaltung und Anwendung der Technik, im Versuch der räumlichen Lösung betriebswirtschaftlicher Probleme in einem liberalisierten Finanzmarktkapitalismus und in einer Informalisierung der Arbeit als de facto Deregulierung. Der Entwicklung der Arbeit in der Plattformökonomie wird zu Recht grosse Aufmerksamkeit geschenkt. Schliesslich kommen bei ihr alle diese Aspekte gemeinsam zum Tragen. Von der gesellschaftlichen Reaktion auf diese nur angeblich technologische Revolution könnte die Zukunft der Erwerbsarbeit abhängen, so Flecker.

Sebastian Schief wiederum fragt sich in seinem Beitrag Towards the Solution of the Economic Problem? - On the Non-Revolutionary Relationship between Working Time and Productivity, warum Keynes' Prognose einer 15-Stunden-Woche bis zum Jahr 2030 mit grosser Sicherheit nicht eintreffen und folglich aus heutiger Sicht eine Arbeitszeit-Revolution nicht eintreten wird. Ausgehend von Keynes' richtiger Einschätzung über die enormen Produktivitätszuwächse, die wir in den letzten hundert Jahren erlebt haben, wagte Keynes die Vorhersage, dass ein gestiegener Lebensstandard uns auf lange Sicht erlauben sollte, unsere Arbeitszeit radikal zu verkürzen. Keynes prognostizierte demnach auch, dass das ökonomische Problem gelöst und die Menschheit sich von ihrer traditionellen Bestimmung lösen könne. Anhand der Beispiele Deutschland, Österreich und der Schweiz zeigt der Beitrag die Entwicklung der Arbeitszeit auf und diskutiert die Frage, warum die Reduktion der Arbeitszeit in den siebziger Jahren abbrach. Der Beitrag schlägt schliesslich ein theoretisches Modell zu Arbeitszeit, Löhnen, Kapital und Produktivität vor.

Sowohl der Beitrag von Amir Sheikhzadegan als auch jener von Daniel Künzler widmen sich schliesslich Revolutionen im politisch-gesellschaftlichen Sinne, wobei Sheikhzadegan die konservative iranische Revolution und Künzler die sozialistische Revolution auf den Seychellen in den Blick nimmt. Amir Sheikhzadegans Beitrag A social revolution in the name of a religion? diskutiert die islamische Revolution im Iran (1978-79) und den starken Einfluss auf die Welt in den folgenden Jahrzehnten. Ein der westlichen Welt wohlgesonnenes monarchisches Regime hatte den Iran unter Zuhilfenahme eines repressiven Sicherheitsapparates einem schnellen Modernisierungsprozess ausgesetzt und wurde durch nationale Proteste gestürzt und durch ein islamistisches Regime ersetzt. Sheikhzadegans Analyse erfolgt aus einer historisch-soziologischen Perspektive und zeigt die Hauptfaktoren der fundamentalen Transformation der iranischen Gesellschaft auf. Der Fokus liegt dabei auf dem dialektischen Zusammenspiel zwischen endogenen und exogenen Faktoren, namentlich imperiale Interventionen, Rohstoff-Ökonomie, soziale Spaltung, Versorgungskrise, radikale Ideologien und die erhebliche Dynamik der schiitischen Gemeinschaft. ${ }^{2}$

Daniel Künzler untersucht in seinem Beitrag The "socialist revolution" in the Seychelles: continuities and discontinuities in economic and social policies die Sozial- und Wirtschaftspolitiken, die in diesem kleinen Inselstaat zu einem vergleichsweise hohen Lebensstandard geführt haben. Nach der „sozialistischen Revolution“ 1977 wurden nicht nur eine universelle Sozialrente, sondern auch kostenlose Schulbildung und Gesundheitsversorgung eingeführt. Die Seychellen haben dabei einen in mehrfacher Hinsicht einzigartigen Weg zu universellen

\footnotetext{
${ }^{2}$ Nicht mehr Gegenstand des Beitrags ist die nachfolgende Kulturrevolution im Iran.
} 
Sozialpolitiken eingeschlagen. Einerseits konnten die Sozialpolitiken durch eine nichtdogmatische Wirtschaftspolitik finanziert werden, welche sich auf drei nur teilweise staatlich gelenkte Schlüsselsektoren fokussierte. Besonders wichtig war, dass die koloniale Plantagenwirtschaft sehr schnell durch Dienstleistungen (Tourismus, Finanzsektor) abgelöst wurde. Andererseits hat die Regierung es auch geschafft, mit einer erfolgreichen Navigation im Kalten Krieg Entwicklungshilfe aus kapitalistischen Staaten zu erhalten und damit die „sozialistischen“ Politiken zu finanzieren. Aussergewöhnlich ist ausserdem, dass die universellen Sozialpolitiken auch nach den ökonomischen und politischen Liberalisierungen weitergeführt wurden.

Verschiedene dieser Beiträge machen deutlich, dass bei allen Diskontinuitäten immer wieder auch Kontinuitäten zu beobachten sind. Wir wünschen Ihnen eine anregende Lektüre!

Sebastian Schief und Daniel Künzler

\section{Literatur}

Allyn, David (2000). Make love, not war. The sexual revolution, an unfettered history. Boston: Little, Brown.

Bell, Daniel (1990). Die dritte technologische Revolution und ihre möglichen sozioökonomischen Konsequenzen. Merkur, 44(1), 28-47.

Childe, V. Gordon (1951). Man Makes himself. London: Mentor Book.

Dahrendorf, Ralf (1990). Reflections on the Revolution in Europe: In a letter intended to have been sent to a gentleman in Warsaw. New York: Random House.

Eisenstadt, Shmuel N. (1978). Revolution and the Transformation of Societies: A Comparative Study of Civilizations. New York: Free Press.

Friedmann, Georges (1936). La crise du progrès. Esquisse d'histoire des idées 1895-1935. Paris: Gallimard.

Gaud, William S. (1968). The Green Revolution: Accomplishments and Apprehensions. Meeting of the Society for International Development, 8 March, 1968. Internet-Site. Zugriff am 07.02.2017 auf http://www.agbioworld.org/biotech-info/topics/borlaug/borlaug-green.html

Goldstone, Jack A. (1982). The comparative and historical study of revolutions. Annual Review of Sociology, 8(1), 187-207.

Goldstone, Jack A. (1986). Revolutions and superpowers. In: Jonathan R. Adelman (ed.). Superpowers and Revolution. New York: Praeger, 38-46.

Goldstone, Jack A. (2001). Toward a Fourth Generation of Revolutionary Theory. Annual Review of Political Science, 4, 139-187.

Grosser, Florian (2013). Theorien der Revolution zur Einführung. Hamburg: Junius.

Huntington, Samuel P. (1968). Political Order in Changing Societies. New Haven: Yale University Press. 
Kraus, Richard Curt (2012). The Cultural Revolution: A Very Short Introduction. Oxford: Oxford University Press.

Kuhn, Thomas S. (2006). Die Struktur wissenschaftlicher Revolutionen (2. revidierte und um das Postskriptum von 1969 ergänzte Aufl., 19. Nachdr.). Suhrkamp: Frankfurt am Main.

Kuran, Timur (1991). Now Out of Never: The Element of Surprise in the East European Revolution of 1989. World Politics, 44(1), 7-48.

Landes, David S. (1983). Der entfesselte Prometheus. Technologischer Wandel und industrielle Entwicklung in Westeuropa von 1750 bis zur Gegenwart. München: dtv.

Lenski, Gerhard Emmanuel, Jean Lenski and Patrick D. Nolan (1991). Human Societies. An Introduction to Macrosociology. Sixth Edition. New York et al.: McGraw-Hill.

Marx, Karl und Friedrich Engels (1848). Manifest der Kommunistischen Partei (Nachdruck 1998). Trier: Karl-Marx-Haus.

Pareto, Vilfredo (1968). The Rise and Fall of the Elites. An application of theoretical sociology. Totowa, N.J.: Bedminster.

Rifkin, Jeremy (1995). Das Ende der Arbeit und ihre Zukunft. Frankfurt am Main: Campus.

Sanderson, Stephen K. (1999). Social Transformations. A general theory of historical development (Expanded edition). Lanham et al.: Rowman \& Littlefield.

Skocpol, Theda (1979). States and Social Revolutions: A Comparative Analysis of France, Russia and China. Cambridge: Cambridge University Press.

Smelser, Neil J. (1959). Social Change in the Industrial Revolution: An Application of Theory to the British Cotton Industry. Chicago: University of Chicago Press.

Sorokin, Pitirim A. (1925). Sociology of Revolution. Philadelphia: J.B. Lippincott.

Tilly, Charles (1996). European Revolutions, 1492-1992. Oxford: Wiley-Blackwell.

Weber, Max (1995). The Russian Revolutions. Ithaca, NY: Cornell University Press. 